\title{
Effets des anabolisants sur les spectres de courants thermostimulés des muscles de veaux
}

\author{
C Lacabanne *1, G Mouthon 2, A Lamure 1, J Dandurand 1 \\ 1 Université Paul Sabatier, laboratoire de physique des solides, \\ 118, route de Narbonne, 31062 Toulouse Cedex; \\ 2 École nationale vétérinaire d'Alfort, laboratoire INRA de la chaire de physique et chimie \\ biologiques et médicales, 7, avenue du Général de Gaulle, 94704 Maisons-Alfort Cedex, France
}

(15é réunion du groupe Développement, INRA, Paris, 24-26 mai 1989)

\begin{abstract}
Résumé - Les auteurs utilisent la méthode de spectroscopie par courants thermostimulés (CTS) pour étudier les caractéristiques d'un tissu musculaire (pectoralis transversus), issu de veaux témoins ou ayant été traités avec des anabolisants (zéranol et trenbolone en implants). Les spectres obtenus sur les 20 échantillons témoins et traités (race Frisonne) montrent des différences significatives. D'autres travaux sont actuellement en cours, afin de permettre l'utilisation de ces observations dans la reconnaissance de viandes provenant d'animaux traités aux anabolisants, et l'identification de l'origine des modifications signalées par cette méthode.
\end{abstract}

anabollsant / pectoralis transversus / veau / spectroscople par courants thermostlmulés

Summary - Effects of growth substances on thermostimulated current spectra from calf muscle. The effects of growth substances (zeranol, trenbolone) on calf muscle (pectoralis transversus) have been studied with a recent physical method (thermostimulated current spectroscopy). This method appears promising for detecting meat from calves treated with such hormonal substances. The authors have hypothesized that the observed differences are related to protein modification, the nature of which is yet unknown. A complementary work is now in progress.

anabollc substance / pectoralis transversus / spectroscopy / calf muscle

\section{INTRODUCTION}

L'utilisation d'hormones anabolisantes en élevage reste difficile à contrôler en fin de cycle de production, à l'abattoir ou à la consommation. Ceci pose un problème d'autant plus aigu que l'usage d'anabolisants doit pouvoir être vérifié, compte tenu de l'interdiction européenne, et en raison des risques potentiels liés aux résidus de ces produits.
Des anabolisants, comme le zéranol ou l'estradiol, associés à l'acétate de trenbolone, laissent peu de résidus et sont difficiles à détecter lorsqu'ils sont utilisés selon les normes du fabricant.

Une détection directe dans la viande implique généralement l'utilisation de méthodes de dosage sensibles dans une gamme de concentration de 0,02-0,06 ng/ g (O'Keefe et Hopkins, 1987). Le recours obligé à des méthodes sensibles mais

\footnotetext{
* Correspondance et tirés à part
} 
lourdes comme I'HPLC (Fukuhara et al, 1986) incite à développer des méthodes plus souples, comme des systèmes de dosage immunoenzymatique, particulièrement des EIA (Meyer et Hoffman, 1987), qui n'évitent pas toujours une étape préalable de préparation, comme l'HPLC.

La mise en évidence des effets des anabolisants sur la composition et la structure des tissus musculaires d'animaux traités n'a pas abouti à une méthode simple et générale de détection. Ainsi, l'étude des modifications du muscle urétral chez les mâles et les femelles (Amara et al, 1987) traités respectivement par l'association zéranol-trenbolone ou par des œstrogènes, apparaît comme une des rares méthodes applicables en expertise, mais reste difficile en pratique. L'étude des modifications biochimiques et structurelles des viandes est utilisable sur des pièces de découpe, et a pu donner certains résultats, cependant inconstants et dépendants du muscle étudié (Kopp et al, 1984; Patterson et Salter, 1985; Canfield et al, 1986; Ouali et al, 1988).

Des méthodes plus particulières comme le typage des isomyosines (Kopp et al, 1984; Ouali et al, 1988), l'analyse "conformationnelle» des protéines par colorimétrie différentielle et l'étude de l'eau liée par RMN, apparaissent prometteuses (Ouali et al, 1988), tout en présentant les mêmes restrictions d'utilisation.

La méthode utilisée ici (spectroscopie par courants thermostimulés) permet d'étudier globalement la polarisation de protéines musculaires et la liaison de l'eau à ces protéines. La sensibilité et le haut pouvoir de résolution de cette technique (plus sensible que l'analyse enthalpique différentielle) nous paraissent adaptés à l'étude d'échantillons de muscles provenant de veaux traités par des anabolisants.
Avant de présenter les résultats obtenus, nous rappellerons brièvement le principe de la technique.

\section{MATÉRIEL ET MÉTHODE}

Le principe de la spectroscopie par courants thermostimulés (CTS) est le suivant : l'échantillon à étudier est placé entre les plaques d'un condensateur où il est soumis à un champ électrique statique $E$, à une température $T_{p}$ pendant un temps $t_{p}$. Le champ $E$ doit permettre à $N$ dipóles de moment $\mu$ de s'orienter. L'échantillon peut acquérir une polarisation $P_{0}$ telle que $P_{0}=$ $N^{2} / 3 K T_{p}$ où $K=$ constante de Boltzmann.

Cette polarisation s'établit avec une constante de temps $\widetilde{\Theta}\left(T_{\rho}\right)$. Le temps de polarisation doit donc être $t_{p}>3 \mathcal{G}\left(T_{P}\right)$, pour que la polarisation $P_{o}$ soit atteinte.

Notons qu'on adopte pour $t_{p}$ et $T_{P}$ les valeurs minimales, afin d'éviter la formation de charges d'espace qui donneraient des pics CTS supplémentaires, susceptibles de polluer le signal intrinsèque. La polarisation est alors gelée en abaissant la température jusqu'à $T_{0} T_{P}$ et le champ $E$ est coupé. Le condensateur est alors branché à l'entrée de l'électromètre. Une élévation contrólée de la température de l'échantillon provoque le retour à l'équilibre des entités polarisables et un courant de dépolarisation est enregistré, en fonction du temps et de la température.

Des pics CTS seront alors observés, dont la position en température est caractéristique des entités polarisables, et décrits sous le nom de "modes".

\section{Dispositif expérimental}

La cellule de mesure de CTS est schématisée en figure 1. Elle comprend:

- une enceinte étanche (1), qui permet d'établir une atmosphère contrôlée d'hélium au niveau de l'échantillon;

- un condensateur plan (2), constitué de 2 électrodes de forme circulaire qui sont reliées 


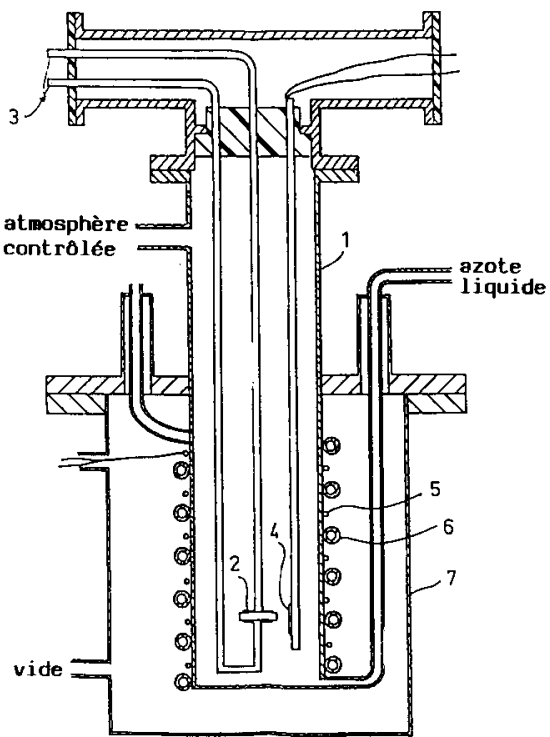

Fig 1. Schéma du dispositif expérimental de mesure des spectres CTS.

par l'intermédiaire des conducteurs (3), soit à la source de tension lors de la polarisation, soit à l'électromètre (Keithley 642) lors de la dépolarisation;

- une sonde de platine (4), mesurant la temperature et branchée, avec la sortie de l'électromètre, à l'entrée d'un micro-ordinateur afin d'établir la courbe de l'intensité du courant en fonction de la température;

- la résistance (5) qui assure le chauffage,

- la circulation d'azote liquide (6) permettant le refroidissement;

- l'enceinte (7) réduit les échanges thermiques extérieurs en maintenant un vide secondaire isolant, autour du système de refroidissement et de chauffage.

Le dispositif permet des refroidissements de $40^{\circ} \mathrm{C}$ par min et des réchauffements de $7{ }^{\circ} \mathrm{C}$ par min, dans une gamme de températures de $-180^{\circ} \mathrm{C}$ à $250^{\circ} \mathrm{C}$. Une description plus détaillée du dispositif expérimental a été présentée par Saadat (1988).

\section{Matériel biologique}

Les fragments musculaires utilisés ont été prélevés dans le muscle pectoralis transversus de 40 veaux de race Frisonne : 20 témoins et 20 veaux traités implantés avec l'association zéranol $(36 \mathrm{mg}$ ) et trenbolone (140 mg), $55 \mathrm{j}$ avant l'abattage (intervenu à l'âge de 4 mois). Les fragments musculaires pesant $5 \mathrm{~g}$ ont été lyophilisés pour que le degré d'humidité de l'échantillon soit inférieur à $20 \%$. La poudre lyophilisée est ensuite comprimée sous forme de disques de $8 \mathrm{~mm}$ de diamètre et $0,5 \mathrm{~mm}$ d'épaisseur.

\section{RÉSULTATS}

Les 2 séries de 20 échantillons (témoins et traités) ont été étudiées. Nous avons observé une bonne reproductibilité des mesures, dans les conditions de polarisation suivantes : $E=8.10^{5} \mathrm{~V} / \mathrm{m} ; T_{P}=50^{\circ} \mathrm{C} ; t_{p}=$ $120 \mathrm{~s}$.

Nous avons reporté en figure 2 le courant de dépolarisation en fonction de la température $T$ pour les échantillons témoins (courbe $A$ ) et traités (courbes $B$ ) respectivement.

On distingue dans les 2 cas, 3 pics CTS qui ont été désignés par $\alpha, \beta, \gamma$ dans l'ordre des températures décroissantes. Pour faciliter la comparaison des résultats, la conductivité dynamique a été normalisée par $\sigma=1 / S E$, où $S$ est la surface de l'échantillon. Les paramètres adoptés pour caractériser les pics CTS sont les coordonnées de leurs maxima : amplitude $\left(\sigma_{m}\right)$ et de température $T_{m}\left({ }^{\circ} \mathrm{C}\right)$ de conductivité dynamique. Le regroupement des résultats est fait dans le tableau I.

\section{DISCUSSION}

On reconnait sur les spectres CTS des échantillons témoins et traités 3 pics distincts, correspondant aux modes $\alpha, \beta$ et $\gamma$. 
Tableau 1. Paramètres des pics de relaxation. Les valeurs moyennes de maxima des températures $T_{m}$ des differents modes ont été établies en effectuant une moyenne des résultats obtenus sur les 20 échantillons étudiés pour chaque série. Compte tenu de l'incertitude sur les valeurs des intensités des pics, nous ne donnons que les valeurs extrêmes des conductivités $\left(\sigma_{m}\right)$.

\begin{tabular}{|c|c|c|c|}
\hline Courbe A & $T_{m}\left({ }^{\circ} \mathrm{C}\right)$ & $\sigma_{m}(\Omega m)^{-}$ & \\
\hline $\begin{array}{l}\alpha \\
\beta \\
\gamma\end{array}$ & $\begin{array}{r}80 \pm 5 \\
25 \pm 5 \\
-100 \pm 10\end{array}$ & $\begin{aligned} 2.10^{-13} & <\sigma_{m \alpha} \\
\sigma_{m \beta} & =\sigma_{m \alpha} \\
10^{-3} \sigma_{m \alpha} & <\sigma_{m \gamma}\end{aligned}$ & $\begin{array}{l}<3.10^{-12} \\
/ 2 \\
<10^{-2} m \alpha\end{array}$ \\
\hline Courbe B & $T_{m}\left({ }^{\circ} \mathrm{C}\right)$ & \multicolumn{2}{|c|}{$\sigma_{m}(\Omega m)^{-1}$} \\
\hline $\begin{array}{l}\alpha \\
\beta \\
\gamma\end{array}$ & $\begin{array}{r}35 \pm 5 \\
-5 \pm 5 \\
-95 \pm 5\end{array}$ & $\begin{aligned} 5.10^{-14} & <\sigma_{m \alpha} \\
\sigma_{m \beta} & =\sigma_{m \alpha} \\
10^{-2} \sigma_{m \alpha} & <\sigma_{m \gamma}\end{aligned}$ & $\begin{array}{l}<3.10^{-13} \\
/ 2 \\
<10^{-1} \sigma_{m \alpha}\end{array}$ \\
\hline
\end{tabular}

Des études antérieures portant sur d'autres tissus et protéines permettent de penser que de tels pics correspondent pour chacun d'entre eux à un mode de réorientation des dipôles lors de l'augmentation contrôlée de la température (Lamure et al, 1983; Saadat, 1988). En particulier, des études sur des tissus conjonctifs (Lamure et al, 1983, 1986) laissent penser que le mode $\gamma$ des pics CTS a pour origine des modifications locales de conformation moléculaire. Le mode $\beta$ des pics CTS peut, par analogie à l'étude de la $\beta$ lactoglobuline (Genieyz, 1985) être associés à une transition "vitreuse" des protéines, dans une gamme de température de $30^{\circ} \mathrm{C}$ à $-5^{\circ} \mathrm{C}$.

Enfin, il a été observé que le mode des pics CTS disparaît pour un grand nombre de protéines si l'échantillon est chauffé audelà d'une certaine température, qui correspond à la température moyenne de dénaturation d'un grand nombre de protéines (Lamure et al, 1983, 1986).

Dans les muscles, les 3 pics CTS appelés $\alpha, \beta, \gamma$, ont donc probablement pour ori- gine de la polarisation des protéines constitutives des échantillons de muscle étudiés. Le décalage des maxima des pics CTS en fonction de la température et selon l'origine des échantillons fait apparaître des modifications liées au traitement hormonal des veaux. Le mode $\gamma$ ne semble pas modifié sur les échantillons ayant subi un traitement hormonal. Par contre, les modes $\beta$ et $\alpha$ sont profondément modifiés, (valeur de $T_{m}$ inférieure de $30^{\circ} \mathrm{C}$ pour le mode $\beta$ et inférieure de $40^{\circ} \mathrm{C}$ pour le mode $\alpha$ ).

En raison des importantes variations d'intensité de ce pic, tant sur les échantillons traités que témoins, l'étude de la relaxation $\alpha$ des pics CTS nous apparaît prometteuse, quant à son utilité pour détecter les tissus musculaires provenant d'animaux traités aux anabolisants. Une telle détection ne pourra alors se faire que sur des échantillons n'ayant pas subi de traitement thermique trop important, car comme en témoigne la figure 2 (courbe A') le pic $\alpha$ disparaît si l'échantillon est chauffé audessus de $90^{\circ} \mathrm{C}$. De plus, l'apparition du 
pic de mode $\alpha$ se situe dans une gamme de températures pour laquelle les mesures se sont montrées les plus reproductibles.

Ces résultats originaux devront naturellement être reproduits sur d'autres muscles que le pectoralis transversus et avec d'autres anabolisants. En effet, les modifications biochimiques ou biophysiques induites par les traitements anabolisants et classiquement décrites dépendent souvent du muscle considéré et de l'âge (Canfield et al, 1986; Amara et al, 1987; Ouali et al, 1988). La méthode utilisée ici pourrait par ailleurs contribuer à mieux connaître les modifications musculaires induites par un traitement anabolisant, difficilement détectables par d'autres méthodes. La nature de ces modifications reste encore à déterminer de façon formelle, et fait l'objet d'investigations en cours.

\section{CONCLUSION}

La méthode de spectroscopie des courants thermostimulés permet de mettre en

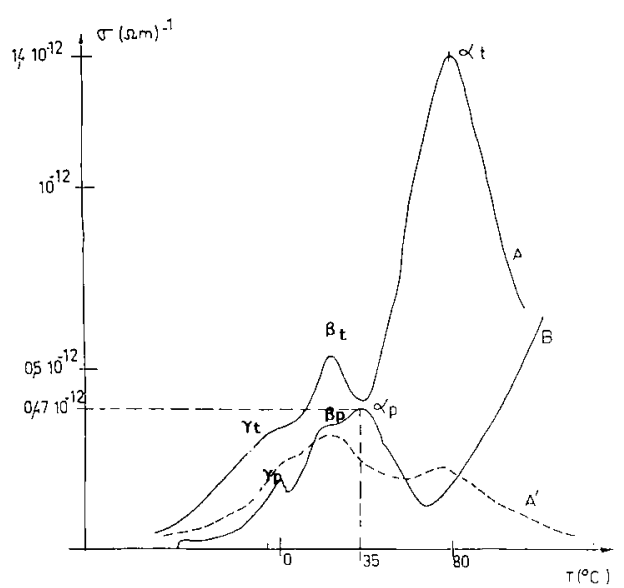

Fig 2. Spectres CTS : viande témoin lors d'une première $(A)$ et d'une deuxième mesure $\left(A^{\prime}\right)$, viande traitée (B). évidence des modifications, probablement de protéines, dans le muscle pectoralis transversus de veaux traités aux anabolisants. Sous réserve d'étendre ces observations à d'autres types d'échantillons pour assurer son caractère prédictif, cette méthode, dans les conditions de l'inspection sanitaire des viandes, pourrait pallier l'insuffisance de certaines analyses toxicologiques.

\section{REMERCIEMENTS}

Nous remercions Mme C Elbaz pour sa collaboration technique.

\section{RÉFÉRENCES}

Amara A, Sautet JY, Van Haverbecke G, Cabanie P, More J, Pavaux C (1987) Anatomical Histochemical and morphometric changes in the musculus urethralis in the female calf under the influence of the association Estradiol-Trenbolone acetate. Anat Histol Embryol 16, 306-319

Canfield LM, Cross HMR, Searle JW, Davy LA (1986) Effects of zeranol on lipid composition of beef Longissimus dorsi. Tex Agric Exp STN Prog Rep (4461-4498), 27-28

Fukuhara K, Matsuki Y, Nambara T (1986) Determination of zeranol and estradiol in beef by High Performance Liquid Chromatography with electrochemical detection. J Food Hyg Soc Jpn 27, 517-521

Genieyz MH (1985) Influence du champ électromagnétique sur la conformation de la protéine béta-lactoglobuline. Thèse de Docteur Ingénieur, ENSAE

Kopp J, Bonnet M, Zabari M, Renou JP, Valin C (1984) Influences relatives du sexe et d'un traitement anabolisant sur les caractéristiques du muscle Long Dorsal de veau. 30th Eur Meet Meat Res Workers. Bristol 9-14 sept 1984

Lamure A, Hitmi N, Harmand MF, Maurel E, Pierraggi MT, Lacabanne C (1983) Etude de vieillissement du collagène par spectroscopie 
diélectrique très basse fréquence. Innov Techn Biol Med 4, 308-327

Lamure A, Hitmi N, Maurel E, Lacabanne C (1986) Polarisation phenomena in collagens from various tissues. IEEE, Trans Electric Insulation, EI, 21, 443-447

Meyer HHD, Hoffman S (1987) Development of a sensitive microtitration plate enzymeimmunoassay for the anabolic steroid trenbolone. Food Addit Contam 4, 149-160

O'Keeffe M, Hopkins JP (1987) Survey of residue concentration of the anabolic agents trenbolone acetate Finaplix and zéranol Ral- gro in beef for the domestic and export market. Ir J Food Sci Technol 11, 13-24

Ouali A, Zabari M, Renou JP, Touraille C, Kopp $\mathrm{J}$, Bonnet M, Valin C (1988) Anabolic agents in beef production effects on muscle traits and meat quality. Meat Sci 24, 151-162

Patterson RLS, Salter LJ (1985) Anabolic agents and meat quality : a review. Meat $\mathrm{Sci}$ $14,191-220$

Saadat AS (1988) Étude de la mobilité moléculaire du collagène par courants thermostimulés. Modélisation du vieillissement cutané. Thèse d'université, université de Toulouse 